Reprod. Nutr. Dévelop., 1988, 28 (1), 207-208.

\title{
Infusion duodénale de lysine et de méthionine et utilisation de l'azote chez l'agneau recevant des régimes contenant du lupin ou du pois
}

\section{R. GUILHERMET, R. TOULLEC}

I.N.R.A., Laboratoire du Jeune Ruminant

et E.N.S.A. de Rennes, 65, rue de St-Brieuc, 35042 Rennes Cedex, France.

Summary. In the growing lamb given maize-base diets containing lupine, a duodenal infusion of lysine + methionine resulted in an increase of nitrogen retention. When the diets contained pea, the infusion of amino acid did not change nitrogen retention.

Chez l'agneau recevant des régimes riches en maïs, des apports postruminaux de méthionine (Mowat et Deelstra, 1972) ou de lysine (Amos, Evans et Burdick, 1980) sont susceptibles d'accroître la rétention azotée. Le but de cet essai était d'étudier l'effet de l'infusion duodénale d'un mélange de lysine et de méthionine chez des agneaux recevant des régimes à base de maïs-grain complété par du pois ou du lupin.

Matériel et méthodes. Le pois et le lupin ont été utilisés à 2 taux différents pour constituer des aliments à niveau azoté haut $(\mathrm{H})$ et bas $(\mathrm{B})$. Les aliments " pois " $\mathrm{B}$ et $\mathrm{H}$ contenaient respectivement 65 et $50 \%$ de maïs, 30 et $45 \%$ de pois et $5 \%$ de complément minéral et vitaminé (CMV); ils renfermaient 12,4 et $14,2 \%$ de $N^{*} 6.25$ par rapport à la matière sèche (MS). Les aliments "lupin " $B$ et $\mathrm{H}$ contenaient 80 et $70 \%$ de maïs, 15 et $25 \%$ de lupin et $5 \%$ de CMV ; ils renfermaient 12,1 et $14,5 \%$ de $N^{*} 6.25 / M S$. Pour chacun des 2 protéagineux, 4 agneaux (Charmois Romanov) Ile-de-France de $20 \mathrm{~kg}$, munis d'un cathéter duodénal, ont été placés en cages à bilans et utilisés selon un dispositif factoriel en carré latin $4 \times 4$ comportant 4 périodes $(7 \mathrm{j})$ de mesure du bilan de l'azote et 4 traitements : niveaux azotés $H$ et $B$ associés à l'infusion duodénale en continu, soit d'un mélange de méthionine et de lysine, soit d'eau distillée. Les régimes ont été distribués en quantité limitée $\left(80 \mathrm{~g}\right.$ de $\left.\mathrm{MS} / \mathrm{kgPV}^{0}, 75 / \mathrm{j}\right)$ comprenant $92 \%$ d'aliment concentré et $8 \%$ de foin de graminée. Les quantités de méthionine infusée avec les niveaux azotées $B$ et $H$ ont été respectivement de 1,8 et $2,0 \mathrm{~g} / \mathrm{kg} \mathrm{MS}$ avec le pois et le lupin; les quantités de lysine infusée ont été de 1,8 et $2,1 \mathrm{~g} / \mathrm{kg}$ MS avec le pois et de 2,6 et $3,0 \mathrm{~g} / \mathrm{kg}$ MS avec le lupin. Ces apports ont été calculés pour amener $3,5 \mathrm{~g}$ de méthionine et $7,5 \mathrm{~g}$ de lysine par $100 \mathrm{~g}$ de protéines digestibles dans l'intestin grêle. Des prises de sang jugulaire ont été effectuées le matin à jeun à la fin de chaque période, pour le dosage de l'urée et des acides aminés (AA) libres plasmatiques.

Résultats et discussion. Pour les deux régimes, la rétention azotée et la teneur en urée du plasma ont été plus élevées $(P<0,05)$ avec le haut niveau azoté. Les teneurs en AA libres plasmatiques n'ont pas été différentes, ce qui indique que seulement une fraction des AA supplémentaires a pu servir à accrô̂tre la rétention azotée, le reste étant mal utilisé, probablement au niveau du 
rumen. Avec les régimes lupin, la rétention azotée a augmenté sous l'effet de l'infusion de lysine et de méthionine $(P<0,05)$, la teneur en urée du plasma ayant eu tendance à ètre plus faible (tabl. 1). Les teneurs plasmatiques de tous les AA essentiels (sauf celles de méthionine et de lysine) et non essentiels libres ont diminué $(p<0,05)$. Les $A A$ les plus réduits ont été les AA ramifiés (surtout la valine), ainsi que la sérine et la glycine. En revanche, avec les régimes pois, l'infusion d'AA n'a eu d'effet ni sur la rétention azotée, ni sur les critères plasmatiques étudiés, en dehors des teneurs en lysine, méthionine et cystathionine libres qui ont été accrues. Le fait que le pois soit plus riche en lysine que le lupin et qu'il ait fourni une part plus importante des protéines pourrait expliquer cette différence de réponse des agneaux ; il est possible aussi que cela ait été dû à une teneur insuffisante des régimes pois en tryptophane.

TABL. 1. - Azote retenu $\left(\mathrm{g} / \mathrm{j} / \mathrm{kg}^{0,75}\right)$ et teneurs plasmatiques en urée $(\mathrm{mg} / 100 \mathrm{ml})$ et en acides aminés libres (AA) fumoles $/ 100 \mathrm{ml}$ ).

\begin{tabular}{|c|c|c|c|c|c|c|c|c|}
\hline \multirow{2}{*}{$\frac{\text { Régime }}{\text { Traitement }(1)}$} & \multicolumn{4}{|c|}{ Lupin } & \multicolumn{4}{|c|}{ Poids } \\
\hline & $\mathrm{HI}$ & $\mathrm{HT}$ & $\mathrm{BI}$ & BT & $\mathrm{HI}$ & HT & $\mathrm{BI}$ & BT \\
\hline Azote retenu $(a, b)$ & 0,61 & 0,52 & 0,49 & 0,42 & 0,50 & 0,49 & 0,45 & 0,44 \\
\hline Urée $(a, c)$ & 38,2 & 40,7 & 31,0 & 35,1 & 40,3 & 38,5 & 32,7 & 32,8 \\
\hline AA essentiels (2) (b) & 112,1 & 131,8 & 117,5 & 129,6 & 119,7 & 119,1 & 120,5 & 124,0 \\
\hline AA non essentiels (b) & 126,5 & 169,5 & 144,7 & 166,0 & 162,7 & 163,5 & 153,0 & 188,0 \\
\hline Lysine $(b, d)$ & 32,8 & 26,1 & 31,0 & 25,1 & 31,9 & 25,2 & 32,0 & 26,6 \\
\hline Méthionine (b, d) & 8,8 & 5,0 & 8,5 & 5,2 & 8,1 & 4,4 & 7,6 & 4,4 \\
\hline Valine (b) & 21,2 & 26,8 & 22,9 & 26,7 & 23,9 & 23,9 & 23,3 & 21,7 \\
\hline Sérine (b) & 8,8 & 11,1 & 9,0 & 11,7 & 12,7 & 12,2 & 11,2 & 13,6 \\
\hline Glycine (b) & 61,5 & 82,8 & 69,5 & 80,0 & 79,7 & 81,1 & 80,1 & 97,5 \\
\hline Cystathionine $(b, d)$ & 1,1 & 0,7 & 1,3 & 0,8 & 1,3 & 0,6 & 1,5 & 0,8 \\
\hline
\end{tabular}

(1) Niveaux azotés : haut (H), bas (B) ; Infusion : d'acides aminés (1), d'eau (T). (2) Lysine et méthionine exclues. $a, b, c, d$ : Effets niveau azoté $(a, c)$ ou infusion $(b, d)$ significatifs $(P<0,05)$ avec le lupin $(a, b)$ ou le pois $(c, d)$.

En conclusion, chez l'agneau, les régimes riches en céréales (maïs-grain), associées au pois, n'apparaissent pas déficients en méthionine et en lysine. En revanche, avec le lupin, un apport supplémentaire de lysine et de méthionine est susceptible d'améliorer l'utilisation de l'azote. Cependant, des études complémentaires sont nécessaires afin de préciser l'importance respective de ces 2 AA, ainsi que l'intérêt d'une supplémentation en tryptophane, notamment dans le cas de l'utilisation du pois. 